\title{
Lack of association between presentation of diarrhoeal symptoms and faecal isolation of Aeromonas spp. amongst outpatients in Hong Kong
}

Although the clinical significance of Aeromonas spp. in extra-intestinal disease is unequivocal (Altwegg, 1999), evidence for their exact causative role in gastrointestinal disorders is still not conclusive (Johnson et al., 1985). Several previous studies have attempted to determine enteric virulence factors in Aeromonas spp., but none has identified any definitive virulence determinant (González-Serrano et al., 2002; Trower et al., 2000). A more recent study in Canada detecting three haemolysin genes, ahh1, asal and aerA, in 121 clinical and 7 reference isolates of Aeromonas demonstrated that genotype 1 ( $a h h 1$; $n=46)$ and genotype 4 (ahh1-aerA; $n=48$ ) were the most common. Genotype 4 isolates were also shown statistically to be more cytotoxic to Vero cell cultures (Wang et al., 2003).

In our Microbiology Division, we applied the Canadian genotyping scheme to the aeromonads isolated from stool specimens received from public outpatient clinics in the territory over a 12 month period for the investigation of gastroenteritis. This was an attempt to ascertain any significant association between the clinical presentation of diarrhoeal symptoms and the organism genotype, together with a number of other microbiological laboratory factors, including species identity and amount of growth on the primary culture plate.

On receipt, the consistency of stool specimens was examined and then classified as formed, soft, watery, and bloody and/or mucous. Bloody, mucous or watery stool specimens were regarded as direct indication of the presence of diarrhoeal symptoms. Routine clinical microbiology laboratory methods were employed for isolation and identification of common bacterial enteric pathogens to the species level (Murray et al., 1999). Specifically, the culture of aeromonads used modified Monsur agar without gelatin (Monsur, 1961). The growth status of bacterial isolates in the primary culture plates was observed, and graded as heavy, medium and scanty. The Pearson chi-square test and the Fisher's exact test, as appropriate, were performed to test the significance of the association between two categorical variables, and $P$ values were two-sided with the level of statistical significance taken as $0 \cdot 05$.

A total of 253 unique Aeromonas strains isolated from 242 faecal specimens received during the period September 2003 to August 2004 were analysed, including 113 (44.7\%) Aeromonas caviae isolates, 25 (9.9\%) Aeromonas hydrophila isolates and
115 (45.5\%) Aeromonas veronii biotype sobria isolates (Table 1). Two Aeromonas isolates each were contained in 11 specimens. Most isolates [196 (77.5\%)] were found as the sole isolates from the specimens, 92 A. caviae isolates, 20 A. hydrophila isolates and 84 A. veronii biotype sobria isolates. The remaining 35 isolates were found at the same time as up to three non-aeromonad co-isolates, in which 32 were detected with one co-isolate in the same specimen. The co-isolates included mainly Campylobacter species (11), Salmonella species (13) and Vibrio species (11).

Most of the isolates [120 (47-4\%)] were obtained from soft stools; $21(8 \cdot 3 \%)$ were from bloody and/or mucous stools, and 25 $(9 \cdot 9 \%)$ were from watery stools.

The majority, $156(61 \cdot 7 \%)$, of the isolates, belonged to genotype 5 and possessed none of the haemolysin genes sought (Table 1). Excluding these isolates, the most prevalent genotype was genotype 2 , carrying asa 1 only, which comprised $79(31 \cdot 2 \%)$ isolates. The asal gene was the most frequently found haemolysin gene among our isolates. No isolates were detected with $a h h 1$ plus asa1 (genotype 3). There was one unique isolate showing the presence of aerA only, which was newly designated genotype 6

Table 1. Distribution of genotypes among the three Aeromonas spp.

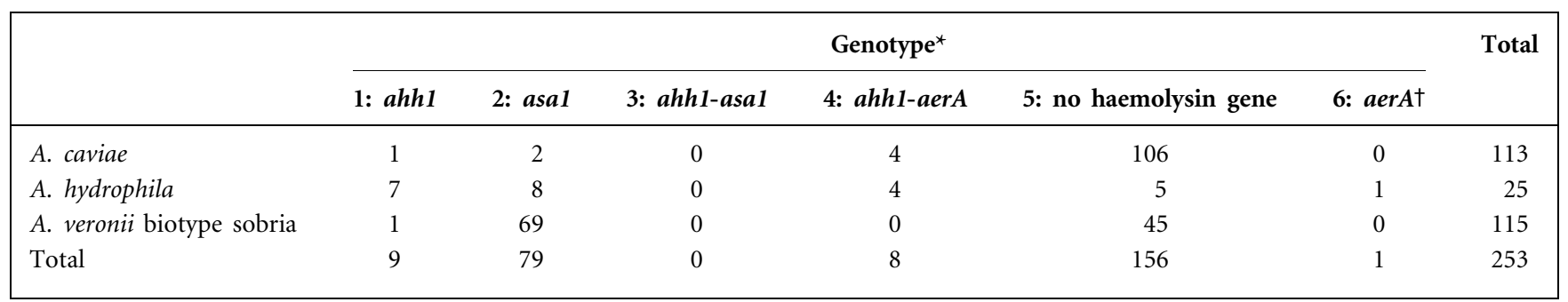

${ }^{\star}$ According to the scheme described by Wang et al. (2003).

tNot in the scheme described by Wang et al. (2003). 
Table 2. Summary of the statistical analyses of the association between the presentation of diarrhoeal symptoms and the various parameters of the Aeromonas isolates in this study

\begin{tabular}{|c|c|c|}
\hline Parameter 1 & Parameter 2 & $P$ value \\
\hline Aeromonas species identity & Number of co-isolates & $0 \cdot 857$ \\
\hline Presentation of diarrhoeal symptoms & Aeromonas species identity & $0 \cdot 306$ \\
\hline Presentation of diarrhoeal symptoms & Genotype 1, ahh 1 & $>0.999$ \\
\hline Presentation of diarrhoeal symptoms & Genotype 2, asa1 & $0 \cdot 220$ \\
\hline Presentation of diarrhoeal symptoms & Genotype 5, no haemolysin genes & $0 \cdot 503$ \\
\hline Presentation of diarrhoeal symptoms & Genotype 6 , aerA & $>0.999$ \\
\hline Presentation of diarrhoeal symptoms & Presence of $a h h 1$ & $0 \cdot 745$ \\
\hline Presentation of diarrhoeal symptoms & Presence of asal & $0 \cdot 220$ \\
\hline Presentation of diarrhoeal symptoms & Presence of aerA & $>0.999$ \\
\hline
\end{tabular}

${ }^{\star}$ For those Aeromonas isolates without co-isolates only.

tChi-square test for linear trend.

and not described in the original scheme (Wang et al., 2003).

Table 2 summarizes the results of the statistical analyses performed. There was no association between the identity of Aeromonas species and the number of co-isolates found $(P=0 \cdot 857)$. The different Aeromonas species found were not related to the occurrence of diarrhoea $(P=0 \cdot 306)$, although those specimens yielding Aeromonas isolates together with co-isolates were more likely to be associated with diarrhoea $(P<0 \cdot 001$, chi-square test for linear trend). However, this should be taken with caution as the co-isolates themselves were well known diarrhoeal pathogens, including vibrios, campylobacters and salmonellae. In fact, it was likely that the diarrhoeal symptoms were due to these co-isolates rather than to the Aeromonas isolates. The distributions of genotypes and haemolysin genes were apparently random among Aeromonas species (Table 1). Notably, in this study in an outpatients setting, the genotypes and the presence of haemolysin genes did not predict the occurrence of diarrhoeal symptoms. Even the presence of virulence factors might not necessarily be phenotypically translated and then clinically expressed. Insufficient evidence for the pathological role of the Aeromonas isolates could thus be obtained. However, the absence of haemolysin genes did not preclude the presence of diarrhoea $(P=0 \cdot 503)$. This study suggests that the causal role of diarrhoea by the described haemolysin genes, and in effect by the Aeromonas spp., is still uncertain.

Even without the influence of co-isolates, different Aeromonas species did not appear to grow in variable amounts on modified Monsur agar primary culture plates $(P=0 \cdot 436)$ (Table 2). Moreover, the growth status of different Aeromonas species on the primary culture plate was not associated with the presentation of patients' diarrhoea $(P=0 \cdot 538)$. This indicates that an increase in the amount of growth of the Aeromonas organisms did not imply a higher chance of diarrhoea. Nevertheless, our study is limited by the possibly incomplete clinical history from the patients. In addition, stool consistency might have normalized by the time the specimens were collected, and hence stool consistency may not be an ideal indicator of clinical illness.

In conclusion, we did not find any significant association between diarrhoeal symptoms presentation and the parameters in our study of aeromonad stool isolates, including bacterial species, genotype, and growth in the primary culture media. Our results suggested that unless we have a definitive genotypic or phenotypic virulence-linked trait as a target, routine faecal culture for Aeromonas spp. is likely to be of limited clinical usefulness. Unfortunately, a definitive trait is yet to be discovered.

Yiu Wai Chu, Chi Ho Wong, Grand K. L. Tsang, Mike S. W. Kwok, Raymond K. O. Wong, Janice Y. C. Lo and Kai Man Kam

Microbiology Division, Public Health Laboratory Services Branch, Centre for Health Protection, Department of Health, 382 Nam Cheong Street, Kowloon, Hong Kong SAR, PR China

Correspondence: Yiu Wai Chu (alf@chp.gov.hk)

Altwegg, M. (1999). Aeromonas and Plesiomonas. In Manual of Clinical Microbiology, 7th edn, pp. 507-516. Edited by P. R. Murray, E. J. Baron, M. A. Pfaller, F. C. Tenover \& R. H. Yolken. Washington, DC: American Society for Microbiology.

González-Serrano, C. J., Santos, J. A., Garcia-López, M. L. \& Otero, A. (2002). Virulence markers in Aeromonas hydrophila and Aeromonas veronii biovar sobria isolates from freshwater fish and from a diarrhoea case. J Appl Microbiol 93, 414-419.

Johnson, P. C., Dupont, H. L., Satterwhite, T. K. \& Wood, L. V. (1985). Lack of correlation between known virulence properties of Aeromonas hydrophila and enteropathogenicity for humans. Infect Immun 50, 62-65. 
Monsur, K. A. (1961). A highly selective gelatin-taurocholate-tellurite medium for the isolation of Vibrio cholerae. Trans $R$ Soc Trop Med Hyg 55, 440-442.

Murray, P. R., Baron, E. J., Pfaller, M. A., Tenover, F. C. \& Yolken, R. H. (editors) (1999). Manual of Clinical Microbiology, 7th edn.
Washington, DC: American Society for Microbiology.

Trower, C. J., Abo, S., Majeed, K. N. \& von Itzstein, M. (2000). Production of an enterotoxin by a gastro-enteritis-associated Aeromonas strain. J Med Microbiol 49, $121-126$.
Wang, G., Clark, C. G., Liu, C., Pucknell, C. Munro, C. K., Kruk, T. M. A. C., Caldeira, R., Woodward, D. L. \& Rodgers, F. G. (2003).

Detection and characterization of the hemolysin genes in Aeromonas hydrophila and Aeromonas sobria by multiplex PCR. J Clin Microbiol 41, 1048-1054. 